\title{
Leçons de l'Asie : grands principes et impératifs mondiaux
}

Lecciones de Asia: grandes principios, imperativos mundiales

Lessons from Asia: Big principles, global imperatives

\section{A. Lin Goodwin}

Traducteur : Jérôme Quintana

\section{(2) OpenEdition}

Journals

Édition électronique

URL : https://journals.openedition.org/ries/4385

DOI : 10.4000/ries.4385

ISSN : 2261-4265

Éditeur

France Education international

Édition imprimée

Date de publication : 4 avril 2015

Pagination : 181-190

ISBN : 9782854206067

ISSN : $1254-4590$

Référence électronique

A. Lin Goodwin, "Leçons de l'Asie : grands principes et impératifs mondiaux », Revue internationale d'éducation de Sèvres [En ligne], 68 | avril 2015, mis en ligne le 04 avril 2017, consulté le 01 juillet 2021. URL : http://journals.openedition.org/ries/4385; DOI : https://doi.org/10.4000/ries.4385 


\title{
Leçons de l'Asie : grands principes et impératifs mondiaux*
}

\author{
A. Lin Goodwin \\ Teachers College, Columbia University, \\ Etats-Unis
}

En l'an 2000, le Programme international pour le suivi des acquis des élèves (PISA) a été mis en place pour la première fois dans 32 pays, pour la plupart membres de l'OCDE (l'Organisation de coopération et de développement économiques). À travers cette évaluation internationale, l'OCDE a permis aux divers pays de se voir proposer un outil commun visant à "vérifier dans quelle mesure les jeunes de 15 ans (...) sont prêts à relever les défis des sociétés d'aujourd'hui » (OCDE/UNESCO-UIS, 2000). Depuis, les comparaisons internationales sont devenues chose courante et les classements PISA s'invitent désormais dans les débats qui se tiennent à l'échelle nationale ou internationale concernant la croissance économique, la qualité des enseignants et les résultats de l'éducation.

Dès le départ, les pays asiatiques ont obtenu des scores fort honorables, figurant parmi les cinq premiers, aux côtés de pays "occidentaux" tels que la Finlande et le Canada. Mais en 2009, les choses ont pris une tournure assez spectaculaire. Shanghai et la Chine ont en effet pris le monde entier par surprise en raflant la première place dans les trois domaines de compétences. D’autres pays asiatiques ont également réalisé de très bons scores, décrochant les cinq premières places dans les trois domaines de compétences, à savoir la compréhension écrite, les mathématiques et les sciences, aux côtés de la Finlande, unique pays non asiatique à occuper le haut du classement. Lors de la dernière enquête PISA, datant de 2012, les pays asiatiques ont figuré en tête du classement, occupant les sept premières places en mathématiques, les cinq premières en compréhension écrite et les quatre premières en sciences. Il n'est guère étonnant que l'OCDE, dans son rapport de présentation des résultats, ait publié la photo de deux élèves asiatiques (sur trois au total) en couverture. Tout le monde semble chercher à percer le secret asiatique et s'interroge pour savoir comment il est possible de parvenir aux mêmes résultats.

Ces excellents scores n'ont toutefois guère suscité l'émoi à Shanghai, peut-être parce que «PISA ne fait que mesurer les performances en compréhension

* Article traduit par Jérôme Quintana. 
écrite, mathématiques et sciences (...). Nous ne pouvons pas dire pour autant que la réussite dans ces domaines de compétences implique la réussite de l'ensemble du système éducatif de base » (Zhang in Tucker, 2014). Ainsi, alors que "les universitaires occidentaux tentent d'identifier certaines politiques et pratiques, de mesurer leurs effets distincts, puis d'affirmer que l'une ou l'autre d'entre elles constitue la réponse à apporter» (Jensen in Tucker, 2014), les universitaires en Chine et d'autres régions asiatiques à forte réussite scolaire ne parlent pas de miracles ni de pratiques meilleures, mais semblent au contraire soucieux de favoriser l'éclosion « d'apprenants de classe mondiale » dotés « d'un désir et d'un potentiel de création et d'innovation, capables de rêver et d'imaginer, de remettre en cause et d'améliorer le statu quo » (Zhao, 2012). Ils sont apparemment attentifs aux problématiques devant lesquelles se trouve notre communauté mondiale et ont conscience que l'aptitude à réaliser de bons scores lors de tests ne permettra pas de résoudre les problèmes du XXI ${ }^{\mathrm{e}}$ siècle et au-delà. Ainsi, si nous voulons tirer de quelconques leçons de "l'Asie », il convient que nous accordions une place moins importante aux résultats des évaluations, que nous nous intéressions moins à certains indicateurs de performances isolés, pris à part, et que nous nous recentrions sur les grands principes qui constituent la base, semble-t-il, de ce que «l'Asie » parvient apparemment à réaliser.

\section{LES LEÇONS DE « L'ASIE »}

Tout d'abord, il est important de déconstruire l'idée que l'on se fait de "l'Asie ", car il existe de multiples régions de l'Asie, aussi diverses que distinctes : l'Asie de l'Est, l'Asie du Sud, l'Asie du Sud-Est, etc. Ces régions abritent ellesmêmes de multiples pays, tous situés en différents points de l'échelle internationale de mesure comparative. L'Asie du Sud-Est, par exemple, inclut le Vietnam, qui a réalisé des scores situés pour la plupart au-dessus de la moyenne des pays de l'OCDE, mais aussi la Thaïlande, qui a obtenu des résultats inférieurs à la moyenne. En outre, les pays d'Asie reposent sur des systèmes économiques, des cultures, des structures sociales et des niveaux de développement tous très différents, ce qui rend plus difficile encore la comparaison entre ces pays et ne permet guère de parler de l'Asie d'une seule et même voix. Cette diversité est d'ailleurs présente non seulement entre mais aussi à l'intérieur de ces pays. C'est la raison pour laquelle ce n'est pas la Chine qui a bondi en tête du classement en 2009, mais la ville de Shanghai, en Chine. De plus, "l'Asie» ne se confine pas aux frontières géographiques de l'Asie, car la diaspora asiatique est très présente à travers le monde. L'Asie est ainsi loin d'être un bloc monolithique et constitue en réalité un phénomène mondial et complexe, qui alimente de nombreuses fausses représentations et de nombreux stéréotypes. La diversité même de cette communauté implique que celle-ci ne peut pas donner lieu à un cadre unique, ni servir de modèle spécifique. 
Dans ce contexte, j'utilise le cas de Singapour à titre d'illustration pour cet article, en m'appuyant sur une analyse récente de quatre systèmes performants (Goodwin, 2014), parmi lesquels celui de Singapour. Ma démarche a consisté à transcender les stratégies spécifiques et les particularités culturelles, afin de faire émerger de grandes idées pouvant apporter un large éclairage sur le sujet. Quelles leçons mon analyse du cas de Singapour a-t-elle donc permis de faire émerger, plus précisément: quelles leçons susceptibles de faire avancer quelque peu les choses et ce, quel que soit le contexte local?

\section{LE CAS dE SingapouR}

Il n'y a pas si longtemps, la simple évocation de Singapour suscitait encore la perplexité. Mais en quelques années, cette perplexité s'est transformée en réel intérêt. On ne s'interroge désormais plus pour savoir de quel pays il s'agit. Cela est dû en partie aux évaluations internationales, qui ont attiré l'attention du monde entier sur les pays très performants comme Singapour, mais en partie aussi à ce que l'on a appelé "le miracle singapourien ", à savoir le passage, en l'espace d'un peu plus d'une génération, d'un pays en voie de développement à un pays totalement développé, financièrement solide, devançant ses concurrents dans ce domaine, un pays qui "continue de figurer en haut de l'échelle économique mondiale " (Versi, 2013). Revenons à peine une génération en arrière : les écoles à Singapour étaient alors jugées de mauvaise qualité ainsi que les enseignants, et les résultats des élèves étaient loin d'être remarquables. Certes, il existait des "poches d'excellence » mais dans l'ensemble, le système éducatif à Singapour ne se distinguait guère par sa qualité. Qu'est-ce qui a permis de changer les choses ? Ce changement est le fruit d'une concertation nationale pour repenser le système éducatif à Singapour, concertation reposant sur la conviction que l'éducation constitue «le principal moteur de l'économie, de la nation et de l'identité " (Luke et al., 2005). En 1997, le Premier ministre de l'époque, Goh Chok Ting, présentait le programme Thinking Schools, Learning Nation (Écoles de la réflexion, pays de l'apprentissage permanent), nouveau projet visant, d'une part, à renforcer les « compétences requises en termes de pensée créatrice et d'apprentissage créateur, afin d'affronter un avenir (...) intensément mondialisé » et, d'autre part, à faire en sorte que l'apprentissage relève de la «culture nationale» (2 juin 1997). L'annonce de ce projet a relancé la politique et la pratique éducatives et constitue la genèse de la refonte du système éducatif singapourien en modèle performant. Une analyse de tous les faits marquants qui ont eu lieu entre cette date-là et l'époque actuelle permet de mettre en lumière deux leçons-clés sur ce qui semble expliquer l'excellence scolaire dans cette république asiatique. 


\section{LEÇON No 1 \\ Priorité nationale : le métier d'enseignant comme profession fondée sur la qualité}

Pour Singapour, l'année 1997 a marqué un tournant dans la reconnaissance du métier d'enseignant comme profession valorisée. Créer une profession fondée sur la qualité nécessite la mise en place de fortes mesures incitatives afin d'attirer les meilleurs candidats, de critères de performance élevés ainsi que d'un curriculum suffisamment large pour préparer les candidats au métier, de prévoir un programme de formation continue en termes de développement professionnel et de rétention du personnel, de permettre l'évolution de carrière et la prise de direction d'établissement ; enfin, de favoriser une culture fondée sur le respect, le statut et le sens. Le gouvernement a commencé par fortement augmenter le salaire des enseignants, mettant ainsi le salaire des nouveaux enseignants à la hauteur de celui des nouveaux diplômés qui intégraient d'autres domaines demandant une préparation et une formation universitaire équivalentes, comme les études d'ingénieur, de droit et de commerce. Cela a permis de conférer à l'enseignement une valeur tangible et d'en faire " une profession de l'apprentissage, comme toute autre profession d'avenir fondée sur la connaissance» (Goh, 1997). Cela a par ailleurs constitué un élément stratégique clé pour attirer des candidats de qualité.

Le gouvernement s'efforce également de soutenir le plus possible les candidats à l'enseignement, lors de leur formation initiale, en prenant notamment en charge la formation, gratuite pour les futurs enseignants, formation qui peut durer jusqu'à deux ans, pendant lesquels ils reçoivent un plein salaire en tant que fonctionnaires du ministère de l'éducation et bénéficient d'avantages divers, notamment d'une prise en charge médicale, ou encore de primes annuelles ou de primes liées aux résultats, et du remboursement de leurs frais matériels, par exemple pour l'achat d'un ordinateur. En retour, les jeunes diplômés s'engagent à exercer dans l'enseignement public pendant trois ou quatre ans. Au-delà de l'aide financière, la formation initiale est complète et met l'accent tant sur le savoir et les contenus que sur la pédagogie. Le curriculum de formation des enseignants fait l'objet d'évaluations régulières de grande ampleur et la philosophie qui sous-tend le programme de formation a été fréquemment réévaluée, révisée et repensée durant la décennie écoulée, avec l'aide de l'organisme TE2 ${ }^{1}$, spécialisé dans le domaine de l'éducation, pour aboutir à la toute dernière forme de conceptualisation d'un modèle propre à la formation initiale des enseignants

1. TE21 = Training and Education in the 21st Century (formation et éducation au XXI siècle). Voir le site web : http://www.te21.com/pages/page.asp?page_id=200168 (NdT) 
(National Institute of Education, 2009). Il n'existe pas de parcours accélérés, de programmes alternatifs ni de voies alternatives d'accès au métier d'enseignant. Lorsqu'il arrive qu'il y ait un manque d'enseignants dans une discipline, cela ne sert pas de prétexte pour se passer de la formation formelle dispensée à l'université. D'ailleurs, la création du National Institute of Education (NIE), comme institut de formation au sein d'un des meilleurs établissements universitaires de Singapour, est l'une des premières mesures prises par le gouvernement pour redorer le blason du métier d'enseignant, et tous les candidats au métier doivent passer par un programme de formation dispensé par l'université. Le ministère de l'éducation et le NIE travaillent en partenariat pour veiller à ce que les établissements scolaires de Singapour aient les enseignants dont ils ont besoin. C'est pourquoi l'offre d'emploi et la formation sont alignés de telle sorte que tous les diplômés du NIE aient la garantie de trouver un travail, le plus souvent dans l'établissement où ils ont effectué leur stage pratique.

Mais pour attirer et retenir les candidats de qualité, il faut aller au-delà des simples mesures incitatives financières et de la formation initiale. Il faut également mettre en place une continuité dans le soutien et l'accompagnement des enseignants, depuis la formation initiale jusqu'à la prise de fonctions, mais également tout au long de leur carrière. Les nouveaux enseignants reçoivent l'aide d'un tuteur et ont un service d'enseignement réduit. Dans le cadre de leur formation, les enseignants bénéficient par ailleurs de 100 heures de développement professionnel, sous la forme de cours ou d'ateliers thématiques. À travers le Teachers' Network (réseau des enseignants), le ministère de l'éducation offre aux enseignants la possibilité (éventuellement à l'aide de bourses) de poursuivre leurs études, ou d'obtenir un congé pour étudier à l'étranger, ou encore d'effectuer un stage dans un établissement proche, dans le but à la fois d'améliorer leurs connaissances, leurs compétences et leurs qualifications, mais aussi de leur redonner de l'élan sur un plan personnel et professionnel. Il existe un système d'avancement de carrière permettant aux enseignants de gravir les échelons selon trois voies ou parcours possibles, et le système d'évaluation qui l'accompagne comprend des primes de résultats fort généreuses. Néanmoins, aucune de ces primes n'est liée aux résultats ou aux performances des élèves. Le système est plutôt conçu pour motiver les enseignants et les inciter à choisir un parcours qui leur permette de progresser dans la carrière. Aujourd'hui, l'accent est mis sur l'accès aux postes d'encadrement, tels que les postes de chefs de département pour les jeunes enseignants (qui ont entre trois et cinq ans à leur actif) manifestant un certain potentiel, et sur l'objectif de $30 \%$ des enseignants au niveau master d'ici 2020.

Enfin, les mesures incitatives d'ordre psychologique sont tout aussi importantes que celles d'ordre financier. On vante les mérites de l'enseignement, qu'on présente comme une profession porteuse de sens, offrant de nombreuses possibilités de carrière. L'éducation représente un enjeu national et on attribue 
aux enseignants un rôle fondamental dans la construction du pays et de son avenir. Les enseignants sont célébrés, respectés, considérés comme étant doués et compétents puisqu'on sait que les recrues font partie des meilleurs (le premier tiers de chaque promotion de diplômés). Lors de chaque discours prononcé à l'occasion de la fête nationale, on intègre des messages sur l'éducation et les enseignants, et les cérémonies annuelles qui marquent la prise de fonctions des enseignants à l'issue de leur formation au NIE comprennent, durant une semaine, des activités auxquelles assistent les hauts responsables, dont le directeur du NIE.

\section{LEÇON No 2 \\ Les enseignants : des professionnels au cœur de l'innovation et du changement}

Lorsque l'on considère l'enseignement comme une profession de haute qualité, les membres de cette profession sont alors traités comme des individus autonomes, capables de réfléchir et de respecter des critères rigoureux de pratique professionnelle. Pour progresser et innover dans n'importe quel domaine, les compétences de professionnels sont indispensables. Ainsi, cette idée selon laquelle il est nécessaire, voire indispensable, que les enseignants soient des professionnels pour améliorer le système éducatif et la réforme éducative est représentative d'un certain nombre de systèmes très performants, dont celui de Singapour. De fait, dans ces pays, la réforme éducative est liée à la recherche sur le métier d'enseignant, et l'obligation qu'ont les enseignants de rendre des comptes relève de leur responsabilité professionnelle.

À Singapour, notamment, l'Academy of Singapore Teachers (AST), créée en 2010 et dirigée par des enseignants, " cherche à bâtir une culture d'excellence professionnelle" (Ministère de l'éducation, 2012). Elle prodigue par ailleurs des conseils et joue un rôle consultatif très important dans le cadre de la réforme éducative. Ainsi, sur le plan de la responsabilité, l'AST a un rôle déterminant dans le développement des compétences des enseignants et dans l'amélioration de leur apprentissage du métier. L'AST a tout d'abord joué un rôle-clé dans le développement du Teacher Growth Model (modèle de croissance de l'enseignant), qui définit « cinq résultats souhaitables pour l'enseignant sur l'ensemble de son parcours professionnel ", en mettant l'accent sur le comportement éthique et compétent, la collaboration, les acteurs de la transformation et le renforcement de la communauté. En outre, l'AST conçoit et propose un large éventail d'activités liées au développement professionnel, notamment un programme en faveur des enseignants débutants, une aide pour les enseignants expérimentés désireux d'obtenir un statut d'enseignant plus élevé, des ateliers dirigés par des enseignants à destination des enseignants et des personnels d'encadrement et de direction. 
L'AST encourage par ailleurs les enseignants de tous les établissements scolaires à s'investir dans des communautés d'apprentissage professionnel qui, de par leur centre d'intérêt, leur rôle ou les thématiques abordées, favorisent l'échange et le débat critique autour des problématiques d'apprentissage et d'enseignement. L'un des objectifs principaux de l'AST est le suivant :

D'une part, favoriser une culture de la recherche, de sorte que les enseignants voient celle-ci comme faisant partie intégrante de leur pratique professionnelle (...) [et] d'autre part, promouvoir la recherche menée par les enseignants, dans le but d'entraîner une amélioration durable et systémique de l'enseignement et de l'apprentissage. (Ministère de l'éducation, 2012).

L'AST aide les enseignants à développer des compétences et des connaissances en matière de recherche, favorise la collaboration entre les enseignants et divers partenaires du ministère de l'éducation et du NIE. Enfin, elle aide les enseignants à obtenir des réponses aux questions qu'ils se posent sur les performances de leurs élèves, à travers la recherche-expérimentation (action-research) et la lesson study, l'étude des cours ${ }^{2}$.

Le pays ayant atteint un effectif stable d'environ 33000 enseignants, la priorité aujourd'hui, à Singapour, est d'accéder à l'étape suivante de professionnalisation du métier d'enseignant et de favoriser l'expertise. Le développement de postes "d'encadrants intermédiaires en milieu scolaire, à savoir des enseignants que l'on repère et que l'on encourage à assumer un rôle important d'encadrement dans leur établissement, sans quitter leur classe » s'effectue selon trois parcours de formation possibles : "via l'université, l'établissement scolaire ou le ministère » (Goodwin, Low et Ng, 2013). Chaque parcours de formation propose une trajectoire différente, mais tous sont conçus pour soutenir, favoriser et utiliser l'expertise des enseignants dans le but d'améliorer les résultats de l'éducation. Les parcours réalisés au sein de l'université renforcent les connaissances et les compétences des enseignants, en proposant davantage de formations post-diplôme sur le plan de la théorie et de la préparation au métier d'enseignant. Les parcours proposés par les établissements scolaires ont pour but de développer les compétences de maîtres-enseignants et de spécialistes de disciplines, afin qu'ils puissent d'une part assumer des fonctions de tutorat et d'accompagnement des jeunes enseignants et des collègues et, d'autre part, superviser l'établissement et la mise en place du curriculum, en devenant personnels d'encadrement ou de direction. Les parcours proposés par le ministère permettent aux enseignants d'assumer périodiquement certaines fonctions au sein du ministère ou du NIE, afin que les praticiens de l'éducation soient intégralement impliqués dans les décisions en matière de politique, de recherche et de curriculum. Il apparaît clairement que Singapour consacre non seulement des ressources importantes à l'encadrement des personnels, mais réfléchit également de manière créative à la

2. Examen rétrospectif des pratiques de l'enseignant dans sa classe. $(\mathrm{NdT})$ 
façon de garantir une large participation et un engagement actif des enseignants confirmés, afin qu'ils puissent assumer des fonctions d'encadrement, contribuer au changement et influencer les résultats et les performances au niveau de l'établissement et/ou du système dans son ensemble.

\section{REMARQUES FINALES}

Dans cette frénésie qui entoure le monde de la comparaison et de la compétition internationale, Singapour et d'autres pays asiatiques progressent à un rythme régulier vers la professionnalisation du métier d'enseignant, en adoptant une approche systémique et globale afin de favoriser le développement, l'amélioration, le soutien et la formation des enseignants et de leur donner les ressources nécessaires à l'accomplissement de leur métier. Singapour peut partager un certain nombre de leçons que le pays a pu tirer de son expérience, mais aucune n'est simple ni facile à mettre en ouvre. Par ailleurs, quelles que soient les leçons, chaque jour qui passe nous amène vers un avenir incertain et relègue certaines " innovations » vers un passé désormais révolu. Ainsi, ce que l'avenir réserve à Singapour, à l'Europe, aux États-Unis ou à tout autre pays demeure une question ouverte. Ce que l'on voit concrètement, ce sont de multiples régions de l'Asie qui, tout à la fois, s'éloignent et se rapprochent de ce que l'on appelle "l'Occident » : elles s'éloignent d'une vision étroite, restreinte et standardisée du curriculum et d'une approche de l'excellence scolaire et de la qualité de l'enseignement fondée sur la punition et la compétition, qui assurément caractérisent de plus en plus certains pays occidentaux comme les États-Unis, pour s'orienter vers des pratiques centrées sur l'élève, intégrées, authentiques, participatives et progressistes, elles-mêmes issues de "l'Occident ». C'est pourquoi il est vain de parler d'un modèle " asiatique ». La mondialisation implique une délimitation moins nette des frontières, des déplacements d'un pays à l'autre, des emprunts, une hybridation. "L'Asie » contre "l'Occident », cela relève d'une fausse dichotomie.

Ce qui est certain, lorsqu'on regarde vers l'avant, c'est que les impératifs du siècle actuel sont de fait de nature mondiale : ce qui survient quelque part dans le monde se répercute ailleurs dans le monde. Non seulement ces impératifs sont loin d'être simples à assumer, mais ils constituent des obligations morales que nous devons tous avoir en tête lorsque nous réfléchissons aux questions liées à l'éducation, à la réussite et au progrès. La première de ces obligations est liée aux disparités hommes-femmes, à l'oppression des femmes et à l'oppression ou à l'exclusion de "l'Autre ", cet " autre » que l'on définit de manière bien trop diverse à travers le monde. La deuxième est liée aux disparités économiques croissantes. La troisième concerne la production exponentielle de connaissances, notamment à travers les technologies numériques. La dernière est liée à la mobilité à l'échelle mondiale. Chacune de ces obligations soulève de nombreuses questions. J'en poserai quelques-unes afin de nourrir le débat. Diversités et 
oppressions : nous sommes tous engagés, du moins de manière rhétorique, dans cette question de l'égalité. Mais qu'est-ce que cela veut dire en termes de changement de la dynamique de pouvoir et en termes de partage des ressources? Sommes-nous disposés à céder nos privilèges afin que d'autres connaissent la justice? Les disparités économiques : c'est effectivement une bonne chose que l'analphabétisme concerne aujourd'hui une part plus faible de la population, mais la réalité est que les individus que l'on considère capables de lire et d'écrire se situent pour la plupart dans un continuum allant de ceux qui font partie de l'élite éducative à ceux qui se contentent de recevoir une éducation très rudimentaire, car la qualité de l'éducation est liée au statut économique : sommesnous prêts à redistribuer les richesses (et si oui, comment?) pour prendre en charge tous les enfants / les enfants des autres ? La production exponentielle de connaissances : le numérique a changé la nature du savoir. Qui est expert, qui a accès à la connaissance, et quels sont les savoirs les plus utiles ? Enfin, concernant cette mobilité sans précédent à l'échelle mondiale : de quelle façon ce phénomène a-t-il modifié l'identité, le nationalisme et la notion de citoyenneté au XXI ${ }^{\mathrm{e}}$ siècle ? Comment les interactions (mondiales) sont-elles (re)façonnées par ce mouvement constant?

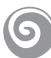

Ces questions ne sont guère abordées car nous nous sommes trop focalisés sur la réussite au détriment de l'apprentissage et sur le capital humain au détriment des êtres humains. Les débats autour de l'enseignement et de l'apprentissage sont centrés sur les aspects économiques et sur les économies émergentes telles que la Chine et l'Inde, ou d'autres pays que l'on a surnommés les «Tigres de l'Asie », en raison de leur puissance croissante. Toutefois, comme l'a dit Albert Einstein : "Tout ce que l'on peut compter ne compte pas forcément; tout ce qui compte ne peut pas forcément se compter». Nous devons nous détourner de la simple comptabilité et nous engager de nouveau à travailler sur ce qui compte vraiment.

\section{BibLIOGRAPHIE}

OECD/UNESCO INSTITUTE OF STATISTICS (2000) : Literacy skills for the world of tomorrow-Further results from PISA 2000. Executive Summary, Paris.

GOH C.T. (1997) : Shaping our future: Thinking Schools, Learning Nation, 7th International Conference on Thinking, Singapour, 2 juin, [en ligne] http://www.moe.gov. sg/media/speeches/1997/020697.htm

GOODWIN A. L. (2014) : "Perspectives on high-performing education systems in Finland, Hong Kong, China, South Korea, and Singapore: What lessons for the U.S.? ", dans S. K. Lee, W. O. Lee, \& E. L. Low (Eds.), Educational Policy Innovations: Levelling Up and Sustaining Educational Achievement (p. 185-200), Singapour : Springer. 
GOODWIN A. L., LOW E. L. et NG P. T. Developing teacher leadership in Singapore: Multiple pathways for differentiated journeys, communication présentée au colloque annuel 2013 de l'American Educational Research Association (AERA), San Francisco.

LUKE A., FREEBODY P., SHUN L., et al. (2005) : " Towards research-based innovation and reform: Singapore schooling in transition ", Asia Pacific Journal of Education, 25(1), p. 5-28.

MINISTRY OF EDUCATION OF SINGAPORE (2012): Academy of Singapore Teachers, [en ligne] http://www.academyofsingaporeteachers.moe.gov.sg/about-ast.

National Institute of Education (NIE) (2009): A teacher education model for the 21 st century, Singapour.

TUCKER M. (ed.) (2014) : Chinese lessons: Shanghai's rise to the top of the PISA league tables, Washington DC : NCEE.

VERSI A. (2013) : "Trading up: South East Asia's economic miracle ", Global: The international briefing, $3^{\mathrm{e}}$ trimestre, p. 56-57.

ZHAO Y. (2012) : World class learners: Educating creative and entrepreneurial students, Thousand Oaks, CA : Corwin. 